\title{
Health Promotion Board-Ministry of Health Clinical Practice Guidelines: Treating Tobacco Use and Dependence
}

\author{
Chan K, Chandler J, Cheong K, Giam PE, Kanagalingam D, Lee JJ, Leong JJ, Ng Y, Oh C, Shi M, Tan ASL, \\ Tan CM, Tan TL, Utravathy $V$
}

\begin{abstract}
The Health Promotion Board (HPB) has updated the clinical practice guidelines on Treating Tobacco Use and Dependence to provide health professionals in Singapore with evidence-based interventions for smoking cessation. This article reproduces the introduction and executive summary of key guideline recommendations (with recommendations from the guidelines) from the HPB-MOH Clinical Practice Guidelines on Treating Tobacco Use and Dependence, for the information of SMJ readers. Chapters and page numbers mentioned in the reproduced extract refer to the full text of the guidelines, which are available from the Health Promotion Board website: http://www.hpb.gov.sg/cpg-smoking-cessation. The recommendations should be used with reference to the full text of the guidelines. Following this article are multiple choice questions based on the full text of the guidelines.
\end{abstract}

\section{INTRODUCTION}

These Clinical Practice Guidelines on Treating Tobacco Use and Dependence are an update of the Smoking Cessation Guidelines developed by the Ministry of Health in 2002. The revised guidelines provide updated evidence-based recommendations to support the effectiveness of interventions to treat tobacco use and dependence. Tobacco dependence is a chronic condition that requires repeated interventions and multiple attempts to quit.

\subsection{Objectives of guidelines}

The aim of these guidelines is to assist all health professionals to identify and assess the tobacco use status of every patient and to deliver evidence-based effective tobacco use and dependence treatments.

\subsection{Target users}

The guidelines are intended for all health professionals, including doctors, dentists, psychologists, pharmacists, dieticians, social workers, occupational therapists, physiotherapists and nurses to assist them in their tobacco use cessation initiatives.

\subsection{Emerging trends of tobacco use \\ 1.3.1 Global trends}

Tobacco use continues to be the leading global cause of preventable death and is a major public health threat to human health. The World Health Organization estimates that about 5 million people die prematurely each year from tobacco-related diseases.

If the current trend continues, by the year 2030 tobacco use will kill more than 8 million people worldwide each year; with $80 \%$ of these premature deaths occurring in low- and middle-income countries. By the end of the century, tobacco will kill a billion people or more unless urgent action is taken.

Tobacco smokers are not only putting themselves at risk but also 1.8 billion non-smokers worldwide. In 2004, it was estimated that $40 \%$ of children, $33 \%$ of male and $35 \%$ of female non-smokers worldwide were exposed to environmental tobacco smoke (ETS).

\subsubsection{Smoking prevalence in Singapore}

The prevalence rate of daily cigarette smoking among Singaporeans has increased from $12.6 \%$ in 2004 to $14.3 \%$ in 2010. In 2010, the smoking prevalence was about 6 times higher among males (24.7\%) compared to females (4.2\%). When compared across the ethnic groups, the proportion of smokers was highest among the Malays (26.5\%), followed by the Chinese $(12.8 \%)$ and Indians (10.1\%). Smoking was also more prevalent among adults aged 18 to 29 years (16.3\%) and adults aged 30 to 39 years $(16.4 \%)$ compared to other age groups (11.4\%-14.5\%).

Between 2004 and 2010 the smoking prevalence rate among the ethnic populations also increased. Among the Malays, smoking prevalence increased from $18.6 \%$ in 2004

\footnotetext{
List of institutions in alphabetical order

Changi General Hospital, College of Family Physicians, Gleneagles Medical Centre, Health Promotion Board, Institute of Mental Health, Khoo Teck Puat Hospital, KK Women's and Children's Hospital, National Healthcare Group, National University of Singapore, Pharmaceutical Society of Singapore, Singapore Armed Forces Medical Corps, Singapore General Hospital

Correspondence: Dr Kenneth Chan, Senior Consultant, Respiratory Medical Associates, Gleneagles Medical Centre, 6 Napier Road \#03-11, Singapore 258499. kpchan@respmed-associates.sg
} 


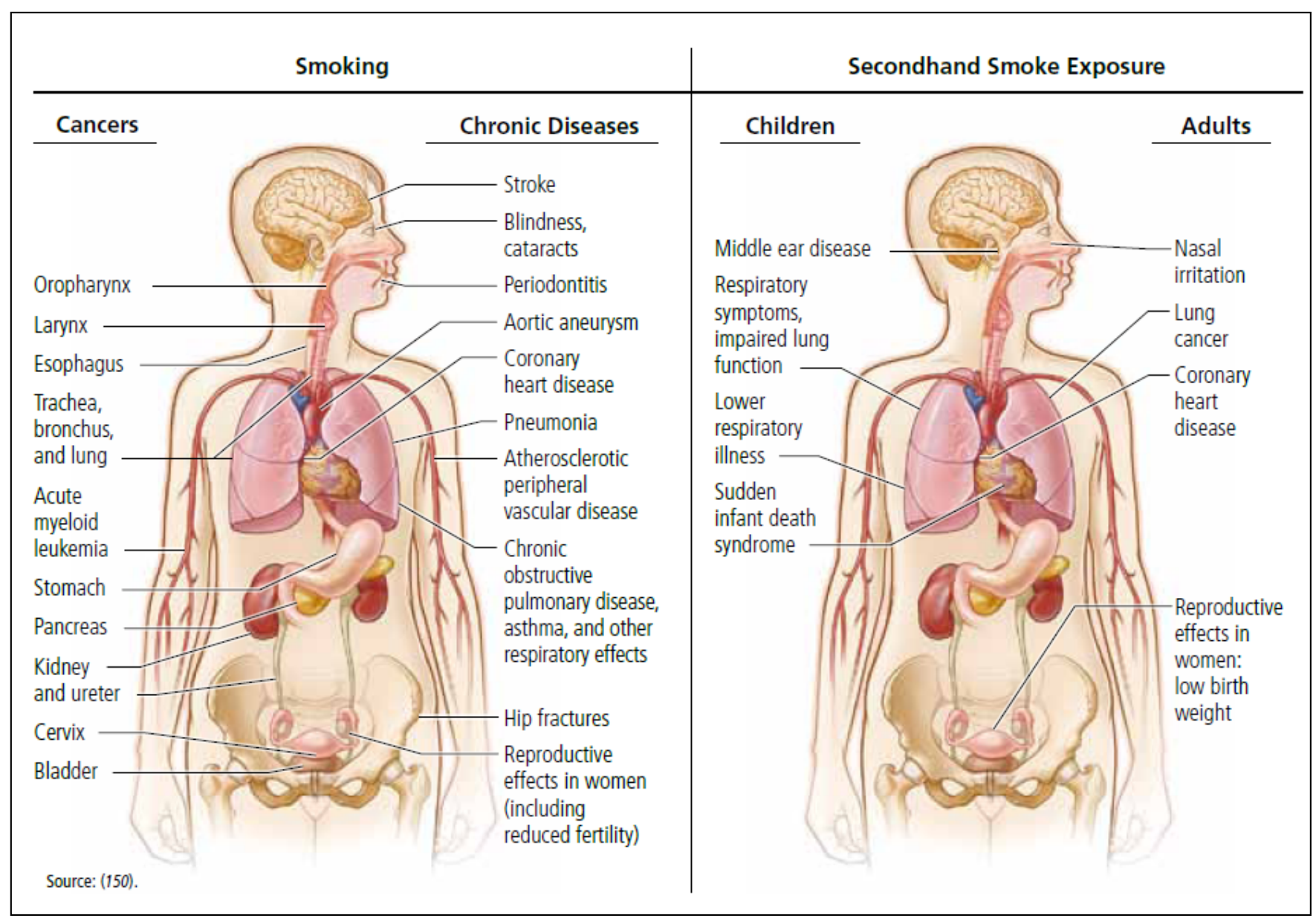

Fig. 1 Harmful effects of tobacco use [WHO Report on the Global Tobacco Epidemic, 2011: Warning about the dangers of tobacco, World Health Organisation. Adapted with permission from: http://whqlibdoc.who.int/publications/2011/9789240687813_eng.pdf. Accessed on 1 November 2011].

to $26.5 \%$ in 2010 . The proportion of smokers aged 18 to 29 years also rose from $18.2 \%$ to $25.3 \%$ among males, and from $6.7 \%$ to $7.3 \%$ among females.

Among students, $6 \%$ of secondary 1 to 4 students smoked at least 1 day in the past 30 days. These students first experimented with cigarettes when they were 13 years old. Social influencers play a part in an adolescent's uptake of smoking, with statistics clearly indicating that adolescents were more likely to pick up smoking if they had a parent, sibling or friend who smoked.

\subsection{Harmful effects of tobacco}

Tobacco use is a major risk factor for many diseases and is also the number one preventable cause of death in the world. On average, a smoker dies 14 years prematurely due to tobaccorelated diseases compared to a non-smoker.

The harmful effects of tobacco use are well documented in numerous studies. Tobacco use harms almost every organ in the human body as shown in Figure 1.

\subsection{Harmful effects of second-hand tobacco smoke}

Second-hand tobacco smoke, also known as environmental tobacco smoke (ETS), is a combination of 'sidestream' smoke (the smoke that is given out by the burning of tobacco product) and 'mainstream' smoke (the smoke exhaled by the smoker).
It is also the tobacco smoke that fills the air in restaurants, workplaces, bars, cars, homes and other enclosed spaces when tobacco users burn tobacco products such as cigarettes, cigars, bidis and shisha/waterpipes.

There are more than 4000 chemicals in tobacco smoke, of which at least 250 are known to be harmful and more than 60 are known to cause cancer. There is no safe level of exposure to second-hand tobacco smoke.

In 2004, approximately 603,000 deaths were attributed to second-hand tobacco smoke worldwide. Globally, exposure to second-hand tobacco smoke has been estimated to have caused 379,000 deaths from ischaemic heart disease, 165,000 from lower respiratory infections, 36,900 from asthma, and 21,400 from lung cancer. Forty-seven percent of deaths from second-hand tobacco smoke occurred in women, $28 \%$ in children, and $26 \%$ in men. It was also estimated that living with a tobacco user increases the non-smokers' risk of developing lung cancer by $20 \%$ to $30 \%$.

\subsection{Third-hand tobacco smoke}

Third-hand smoke first appeared in medical literature in 2009 and it refers to residual tobacco smoke that remains on indoor surfaces after the cigarette has been extinguished. This residue can react with common indoor air to produce dangerous cancer-causing carcinogens. It includes ultrafine chemical 


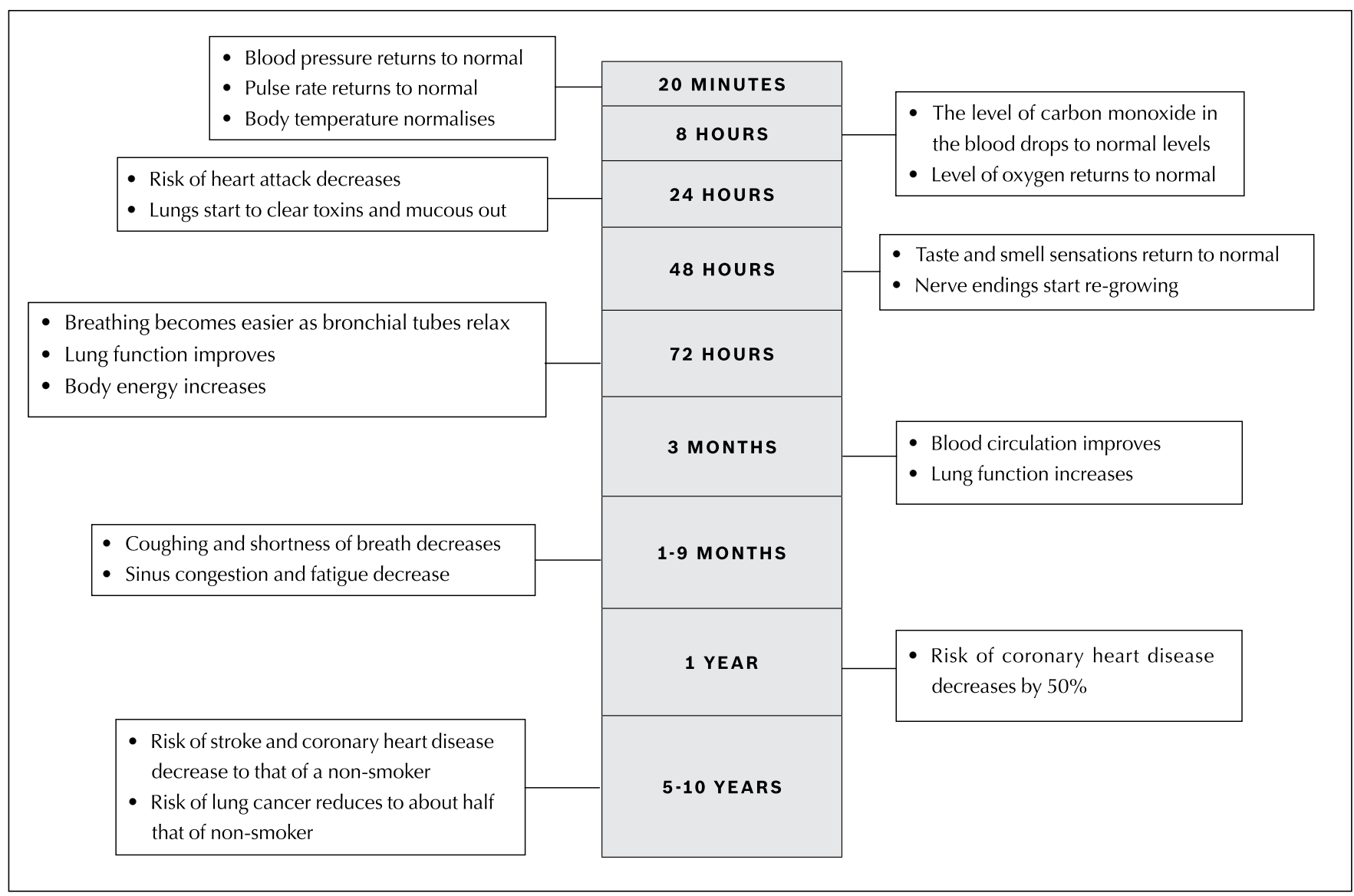

Fig. 2 Health benefits of quitting tobacco use [adapted from Health Promotion Board: http://www.hpb.gov.sg/hpb/default.asp?pg_id=2564].

particles and volatile organic compounds, some of which are known to be toxic. Absorption of these particles could either occur through respiration, ingestion or through the skin.

Third-hand smoke is of concern particularly among young children living in tobacco-using households.

Although there appears to be an increasing interest and research on this, current evidence on its effects on health is still not well documented.

\subsection{Harmful effects of other forms of tobacco products}

All forms of tobacco are harmful and addictive. There is no safe tobacco product. Other forms of tobacco products include smokeless tobacco (also called chewing tobacco, snuff), pipes, cigars, rolled tobacco, shisha/waterpipes, bidis and others.

Cigar users are at risk of cancers of the lung, oral cavity, larynx and oesophagus. Heavy cigar smokers who inhale deeply are also at increased risk of coronary heart disease and chronic obstructive pulmonary disease. Similarly, smokers who smoke pipes are at higher risks of coronary heart disease and cerebrovascular disease when compared to nonsmokers. Studies also showed that shisha/waterpipes contain similar harmful chemicals as cigarettes and are as addictive as cigarettes.

The use of other forms of tobacco products has become more prevalent especially among the adolescents globally. In Singapore, among secondary 1 to 4 students who reported smoking at least 1 day in the past 30 days, 28.8\% indicated using other forms of tobacco products such as shisha/ waterpipes, chewing tobacco, bidis and others. This will be an area of concern if the prevalence of using other forms of tobacco product increases.

\subsection{Benefits of quitting}

Quitting tobacco use has major and immediate health benefits for both men and women of all ages. It is also well established through numerous scientific studies that quitting tobacco use improves health and quality of life almost immediately. The benefits can start after 20 minutes of quitting tobacco use (see Figure 2).

The benefits of quitting tobacco also apply to those who quit at an older age. For example, a healthy man aged 60 to 64 years who smokes a pack of cigarettes or more a day reduces his risk of dying from tobacco-related diseases by $10 \%$ for the next 15 years if he quits tobacco use. After 10 years of abstinence from tobacco, his risk of lung cancer is reduced by about $30 \%$ to $50 \%$. This risk continues to decline with further abstinence from tobacco use.

For patients with pre-existing medical conditions (e.g. ischaemic heart disease), quitting tobacco use is associated with a substantial reduction in risk of mortality. The risk is reduced by approximately $36 \%$ which is much greater compared with other secondary preventive interventions such as lowering cholesterol which has received greater attention in recent years. 
Furthermore, pregnant women who quit tobacco use before the second trimester of pregnancy can reduce their risk of having a low birth weight baby to that of a woman who has never smoked. Stopping smoking also slows the rate of loss of lung capacity in chronic airways disease.

\section{EXECUTIVE SUMMARY AND KEY GUIDELINE RECOMMENDATIONS Executive Summary}

Treating tobacco use and dependence is an important public health goal that will improve the quality of care and the health of all Singaporeans. The primary goal of the clinical practice guidelines is to reduce the prevalence of tobacco use and dependence through cessation treatments.

There is strong evidence to show that tobacco use causes many chronic diseases such as ischaemic heart disease, stroke, multiple cancers, respiratory diseases and complications during pregnancy, that affect the population and the health delivery system.

This updated Clinical Practice Guidelines on Treating Tobacco Use and Dependence is developed to act as a resource and guide for all health professionals to identify and screen tobacco users, to deliver evidence-based tobacco use cessation treatments for patients and specific population groups who use tobacco. Specific populations include hospitalised and pre-operative patients, adolescents, pregnant women, and patients with psychiatric disorders, cardiovascular diseases or alcohol abuse disorder. The guidelines are developed based on comprehensive literature reviews on recent evidence on tobacco use and dependence treatments.

A suggested framework for treating tobacco use and dependence has also been developed to provide a simple step-by-step approach that all health professionals can use. The important message to every health professional is to make treating tobacco use and dependence a priority during the patient's visit. It is important that you ask your patient two key questions:

a) "Do you smoke?" b) "Do you want to quit?" - followed by the use of the recommendations as listed in the various sections of the guidelines.

\section{Key Guideline Recommendations}

Details of recommendations can be found in the main text at the pages indicated.

\section{Non-pharmacological interventions}

A Different forms of person-to-person behavioural support including individual, group and telephone support may be used as interventions to treat tobacco use and dependence. (pg 20)

Grade A, Level 1+
A If resources are available, telephone support provided separately or as part of tobacco use and cessation intervention, should be offered to smokers undergoing a quit attempt. (pg 20)

Grade A, Level 1+

A Where feasible, health professionals should provide multiple behavioural support sessions to treat tobacco use and dependence; with each session lasting more than 10 minutes. (pg 21)

Grade A, Level 1+

B Behavioural interventions such as motivational interviewing or cognitive behavioural therapy may be used to help tobacco users quit. (pg 23)

Grade B, Level 2++

B Technologies such as individual-tailored web-based interventions, or motivational interviewing mobile phone text messages may be considered for tobacco users attempting to quit. (pg 24)

Grade B, Level 2+

A Acupuncture or hypnotherapy is not recommended routinely for reducing tobacco use and dependence. (pg 25)

Grade A, Level 1++

A Whenever possible, both behavioural support and medication should be provided to tobacco users who have the intention to quit tobacco use. (pg 26)

Grade A, Level 1++

\section{Pharmacotherapy interventions}

A All tobacco users who are trying to quit should be offered both behavioural support and medication unless there are contraindications or insufficient evidence of effectiveness in specific populations (i.e. pregnant women and adolescents). (pg 27)

Grade A, Level 1+

B High-dose nicotine replacement may be considered for tobacco users with persistent cravings and withdrawal symptoms. (pg 28)

Grade B, Level 2++

A Eight to twelve weeks of Nicotine Replacement Therapy (NRT) is recommended for most tobacco users undergoing a tobacco use quit attempt. (pg 29)

Grade A, Level 1+ 
A Both bupropion SR and varenicline may be used in conjunction with behavioural support for patients attempting to quit. (pg 30)

Grade A, Level 1++

A Nicotine patches may be combined with another form of NRT or bupropion SR to increase tobacco use abstinence. (pg 31)

Grade A, Level 1+

C E-cigarettes should not be used or prescribed as smoking cessation aids. (pg 32)

Grade C, Level 3

Specific Populations: Hospitalised and pre-operative patients

A Where resources are available, behavioural support should be offered by a trained advisor for tobacco use and dependence to all hospitalised patients who are tobacco users. (pg 34)

Grade A, Level 1++

B NRT should be considered for hospitalised patients who are tobacco users and attempting to quit. (pg 34)

Grade B, Level 1+

A If resources are available, intensive behavioural support interventions for tobacco use and dependence, including the use of NRTs, should be offered to patients over a period of 4 to 8 weeks prior to surgery. (pg 35)

Grade A, Level 1+

\section{Specific Populations: Adolescents}

A All health professionals should provide brief but tailored advice on quitting tobacco use to adolescents who use tobacco. (pg 36)

Grade A, Level 1+

A Pharmacotherapy, including the use of NRT, should not be used routinely in adolescent tobacco users attempting to quit. (pg 36)

Grade A, Level 1++

\section{Specific Populations: Pregnant women}

B Pregnant women who use tobacco should be offered person-to-person behavioural support intervention as the first-line approach to treat tobacco use and dependence. (pg 38)

Grade B, Level 1+

A Pharmacotherapy interventions should not be routinely used for pregnant women attempting to quit. (pg 38)

Grade A, Level 1+

Specific Populations: Patients with psychiatric disorders

GPP NRTs should be used as the first-line treatment for persons with psychiatric disorders. (pg 39)

GPP

GPP Psychiatric patients who are undergoing a quit attempt need to be monitored for adverse effects because of significant interactions between nicotine, pharmacotherapy for smoking cessation and common psychiatric drugs. (pg 39)

GPP

Specific Populations: Patients with cardiovascular disease

A In patients with a recent acute coronary syndrome, NRT may be started just before hospital discharge to assist tobacco users attempting to quit. (pg 41)

Grade A, Level 1+

A Varenicline or bupropion SR may also be used, in combination with behavioural interventions, for cardiovascular disease patients who use tobacco. (pg 41)

Grade A, Level 1+

\section{Specific Populations: Patients with co-morbid alcohol} abuse

B All alcohol dependent patients who are also tobacco users, including those undergoing alcohol addiction treatment programmes, should be offered treatment for tobacco use and dependence. (pg 42)

Grade B, Level 2+ 


\section{SINGAPORE MEDICAL COUNCIL CATEGORY 3B CME PROGRAMME} (Code SMJ 201307C)

These questions are based on the full text of the guidelines, which may be found at http://www.hpb.gov.sg/cpg-smoking-cessation.

Question 1. Regarding tobacco use and quitting:

(a) The harmful effects of tobacco use can also be attributed to second-hand tobacco smoke (i.e. Environmental Tobacco Smoke).

(b) Studies show that waterpipe or shisha is less harmful and less addictive than cigarettes.

(c) Patients with pre-existing medical conditions such as ischaemic heart disease also benefit from quitting.

(d) Almost all the nicotine from tobacco use is metabolised in the body within one month of cessation.

Question 2. Regarding tobacco use and dependence interventions:

(a) As a first line intervention, all health professionals should use the ABC approach - "Ask, Brief advice, Cessation support".

(b) Combination of counselling and medication is the most effective intervention for tobacco use cessation.

(c) For pregnant women, the first line tobacco use cessation intervention should be behavioural support.

(d) Psychiatric patients experience deterioration in their condition if they quit smoking.

Question 3. Pertaining to patients who use tobacco and are about to undergo surgery:

(a) Smokers undergoing elective surgery are at increased risk of postoperative complications when compared to non-smokers.

(b) Intensive tobacco dependence intervention prior to surgery increases the rate of smoking cessation by about ten-fold.

(c) Brief advice regarding smoking may increase abstinence rates compared to no advice but does not reduce the risk of post-operative complications.

(d) The optimal timing for stopping tobacco use is about two weeks prior to surgery.

Question 4. Regarding tobacco use cessation in adolescents:

(a) Nicotine Replacement Therapy (NRT) is not routinely recommended for adolescents.

(b) Health professionals should take into account adolescents' preparedness to quit smoking for effective intervention.

(c) In Singapore, the average age of smoking initiation is 13 years old.

(d) Pharmacological interventions have significantly improved abstinence rate in adolescents who wish to quit smoking.

Question 5. Regarding alcohol and tobacco use:

(a) Alcohol abusers are 2 to 3 times more likely to smoke.

(b) Patients on alcohol dependence programmes are not interested in tobacco use cessation.

(c) Participants in smoking cessation programmes are more likely to abstain from alcohol and other drugs.

(d) Alcoholism does not negate smoking cessation attempts.

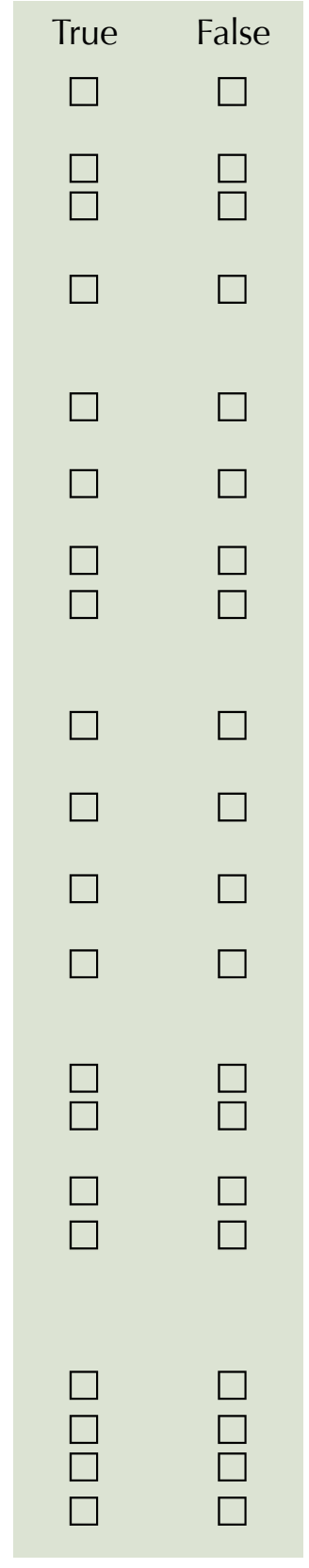

\footnotetext{
Doctor's particulars:

Name in full

MCR number

Specialty:

Email address

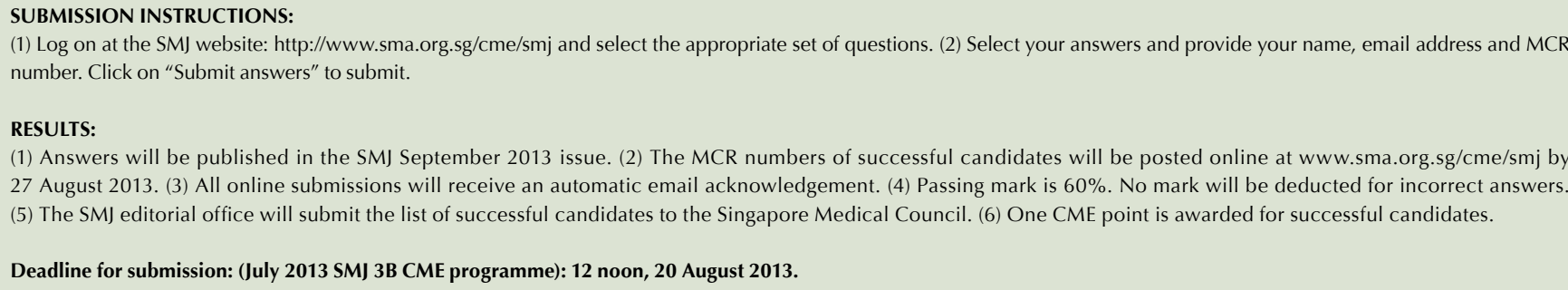

Deadline for submission: (July 2013 SMJ 3B CME programme): 12 noon, 20 August 2013. 